ISSN 1991-8631

Original Paper

http://indexmedicus.afro.who.int

\title{
Caractérisation écologique et structurale des parcs à néré [Parkia biglobosa (Jacq.) R. Br. Ex G. Don] du département de la Donga au Nord-Ouest du Bénin
}

\author{
Kourouma KOURA*, Essè Florentin DISSOU et Jean Cossi GANGLO \\ Laboratoire des Sciences Forestières, Faculté des Sciences Agronomiques, Université d'Abomey-Calavi, \\ 01 BP 526 Cotonou, Bénin. \\ *Auteur correspondant, E-mail : kourakourouma@yahoo.fr; Tél : +22996716130
}

\section{RESUME}

Une étude phytosociologique de la végétation dans les parcs à néré a été réalisée selon l'approche synusiale dans le département de la Donga au Nord-Ouest du Bénin. Le nombre total d'espèces des synusies identifiées varie de 2 à 105. L'indice de Shannon est compris entre 0,02 et 5,08 alors que le coefficient d'équitabilité de Pielou varie de 0,02 à 0,76. L'étape phytocoenologique a consisté à réunir les synusies identifiées sur la base de leurs relations spatio-temporelles en cinq phytocoenoses: phytocoenoses à Pennisetum pedicellatum; à Imperata cylindrica et Andropogon gayanus; à Daniellia oliveri; à Daniellia oliveri et Pteleopsis suberosa; à Hymenocardia acida et Terminalia avicennioides. La densité de ces phytocoenoses varie de 39 à 70 pieds/ha et la surface terrière de 4,93 à 12,38 $\mathrm{m}^{2} / \mathrm{ha}$. Ces valeurs sont fortement liées au mode de gestion des pieds de néré dans la zone d'étude. L'analyse de variance du diamètre de l'arbre de surface terrière moyenne a montré qu'il varie d'une phytocoenose à l'autre. Les structures en diamètre de l'espèce montrent une insuffisance dans sa régénération. Des activités de plantation ou d'enrichissement en néré sont indispensables pour assurer la gestion durable des parcs à néré dans la zone d'étude.

(C) 2013 International Formulae Group. All rights reserved.

Mots clés: Phytosociologie, phytocoenoses, néré, structure en diamètre, Bénin.

\section{INTRODUCTION}

Le milieu forestier regorge d'importantes richesses naturelles diversifiées, que l'homme a presque toujours su mettre en valeur pour assurer sa survie (Mukerji, 1995). Mais parmi toutes ces richesses potentielles que regorgent les forêts, le bois demeure encore le plus reconnu et le plus valorisé (Wong et al., 2001). Pourtant, à part le bois, le milieu forestier fournit une autre catégorie de produits non négligeables, connus sous le nom de Produits Forestiers Non Ligneux (FAO,
1999), qui jouent un rôle très important dans l'amélioration des conditions de vie humaine, en tant qu'aliments, produits médicinaux, matériaux de construction, éléments culturels, etc. Ces Produits Forestiers Non ligneux occupent une place particulièrement importante dans les milieux ruraux africains, où les populations, au pouvoir d'achat souvent très faible, sont constamment exposées aux crises alimentaires (Eyog-Matig et al., 1999 ; Bikoué et Essomba, 2007). Au Bénin, environ 162 espèces végétales sont consommées par 
les populations rurales pour leurs feuilles, leurs fruits, leurs graines, leurs racines, leurs tubercules et leurs fleurs (Codjia et al., 2003). $\mathrm{Au}$ nombre de ces espèces figure le néré [Parkia biglobosa (Jacq.) R. Br. Ex G. Don] dont les fruits sont utilisés par les populations. Il constitue l'une des essences d'intérêt socioéconomique les plus valorisées par les populations locales du Nord-Bénin (Baco, 2007). Parmi les 31 espèces ligneuses médicinales les plus utilisées dans la médecine traditionnelle béninoise, cette espèce occupe la cinquième position (EyogMatig et al., 1999). En outre, de nombreux travaux ont confirmé sa grande valeur nutritive et la place importante qu'il occupe dans l'alimentation au Bénin (Eyog-Matig et al., 2002 ; Gutierrez, 2000 ; Djakpo, 2005). La pulpe de ses gousses est riche en saccharose et ses graines sont riches en glucides, protides, lipides constituant ainsi un excellent aliment énergétique (Djakpo, 2005). Dans la liste des 18 espèces ligneuses alimentaires prioritaires à conserver, établie par la FAO en 2001, il occupe le quatrième rang. Il est aussi une espèce agroforestière importante (Bonkougou, 1987 ; Lafleur, 2008). D'importants travaux de recherche ont été réalisés sur cette espèce en Afrique, surtout dans la sous région ouestafricaine et particulièrement au Bénin. La place et les rôles de cet arbre dans la vie quotidienne des communautés rurales sont davantage connus (Ouédraogo, 1995 ; Guttierez, 2000 ; Sadji, 2000 ; Walla, 2001 ; Koura, 2003). En Afrique de l'Ouest, certaines connaissances biologiques ont été précisées notamment en matière de répartition des peuplements, de phénologie, de système de reproduction, de multiplication végétative, de biologie et d'écologie de la reproduction (Ouédraogo, 1995 ; Sina, 2006). La diversité intra et inter-population, la structure et les distances génétiques entre les différentes populations ont été déterminées (Sina, 2006). Au Bénin, la caractérisation morphologique et structurale des parcs à néré des régions de Bohicon, Savè, Parakou, Bembérèkè, Kandi, a été faite de même que l'évaluation de la production de fruits des pieds de néré de ces parcs (Gbédji, 2003). La dynamique physicochimique et microbiologique de la fermentation des graines de néré pour la production de l'afitin, de l'iru et du sonru (noms locaux de condiments issus de la fermentation des graines de néré) a été déterminée (Azokpota et al., 2006). Des composés volatiles aromatiques ont été également identifiés dans ces condiments dans le but d'obtenir des produits de caractéristiques aromatiques, en fermentation contrôlée, qui répondent à la demande des consommateurs. Malgré tous ces travaux, les communautés végétales qui abritent les parcs à néré du département de la Donga (une des zones de prédilection du néré au Bénin) n'ont pas encore été identifiées. C'est ce qui justifie la pertinence de la présente étude intitulée : «Caractérisation écologique et structurale des parcs à néré [Parkia biglobosa (Jacq.) R. Br. Ex G. Don] du département de la Donga au Nord-Ouest du Bénin ». Cette étude a pour objectif général de contribuer à la gestion durable des ressources agroforestières du département de la Donga. Elle permettra de façon spécifique de: (1) identifier les communautés végétales qui abritent les parcs à néré de ce département et (2) préciser quelques caractéristiques écologiques et structurales de ces communautés végétales.

\section{MATERIEL ET METHODES Milieu d'étude}

Le département de la Donga s'étend sur une superficie de $11126 \mathrm{~km}^{2}$ et est globalement compris entre $8^{\circ} 27^{\prime} 43^{\prime \prime}$ ' et $10^{\circ}$ 01' $57^{\prime}$ ' de latitude Nord et entre $1^{\circ} 20^{\prime} 42^{\prime}$ ', et $2^{\circ} 15$ ' 39', de longitude Est (Figure 1). Il comporte quatre communes que sont : Bassila, Copargo, Djougou et Ouaké. Le régime pluviométrique est celui des climats tropicaux 
de type soudano-guinéen caractérisé par une saison sèche (Novembre à Mars) et une saison pluvieuse (Avril à Octobre). Les sols dominants du département sont ferrugineux tropicaux mais on y rencontre aussi des sols ferralitiques. La végétation est dense le long des cours d'eau et constitue des forêts galeries. Les forêts classées occupent plus de la moitié de la superficie de la commune de Bassila $\left(2437 \mathrm{~km}^{2}\right)$ et une partie de la commune de Djougou où l'on rencontre la savane arborée et la savane arbustive. La flore caractéristique de ce type de végétation est constituée particulièrement de plusieurs espèces ligneuses [Parkia biglobosa, Vitellaria paradoxa Gaertn. f., Tamarindus indica L., Piliostigma thonningii (Schumach.) Milne-Redh., Hymenocardia acida Tul., Pteleopsis suberosa Engl. et Diels, Anogeissus leiocarpus (DC.) Guill. et Perr., Daniellia oliveri (Rolfe) Hutch. et Dalz., Ficus sp. L., Terminalia avicennioides Guill. et Perr., etc.]. Le reste du département est dominé par des savanes (arbustive, herbacée, arborée) où se pratiquent d'intenses activités agricoles. Les espèces telles Parkia biglobosa, Vitellaria paradoxa, Adansonia digitata L., Borassus aethiopum Mart., Blighia sapida Koenig, etc. sont épargnées dans les agglomérations, autour des concessions.

En 2002, le Recensement Général de la Population et de l'Habitat (RGPH) a estimé la population du département de la Donga à 350062 habitants répartis au sein de 44945 ménages. Les ethnies dominantes de cette population sont: Yom, Lokpa, Dendi, Nago, Anii, Foodo, Fulani (INSAE, 2004). On y rencontre également d'autres ethnies provenant des départements limitrophes ainsi que du Togo voisin.

\section{Etude phytosociologique de la végétation}

Afin de décrire dans des détails pertinents les conditions stationnelles dans lesquelles les peuplements de néré se développent, une étude phytosociologique a été effectuée selon l'approche synusiale intégrée (Gillet et al., 1991; Gillet, 2000) dans trois communes du département de la Donga (Djougou, Bassila et Ouaké). Les relevés phytosociologiques ont été faits au sein de trente (30) placeaux de formes et de superficies variables (entre 0,0579 ha et 0,99 ha), mis en place pour collecter les données dendrométriques sur le néré. Ces placeaux contiennent au moins 10 pieds de Parkia biglobosa (Duplat \& Perrote, 1981). Le détail de la méthode phytosociologique utilisée est résumé dans Ganglo et De Foucault (2006).

\section{Méthode d'étude des paramètres dendrométriques}

La circonférence à $1,3 \mathrm{~m}$ du sol de tous les arbres de néré de chaque placeau a été mesurée à l'aide d'un mètre ruban.

Certains paramètres dendrométriques ont permis de caractériser les phytocoenoses identifiées. Il s'agit notamment du diamètre de l'arbre de surface terrière moyenne $\left(\mathrm{D}_{\mathrm{g}}\right)$, de la surface terrière $(G)$ et de la densité de peuplement.

Nous avons calculé le diamètre de l'arbre de surface terrière moyenne $\left(\mathrm{D}_{\mathrm{g}}\right)$ par la formule suivante :

$\mathrm{D}_{\mathrm{g}}=\sqrt{\frac{\sum d i^{2}}{n}}$

avec $d i$ le diamètre en $\mathrm{cm}$ (1)

de l'arbre i du placeau considéré et $n$ le nombre d'arbres dans le placeau.

La surface terrière $(G)$ est la somme de la surface des sections transversales des individus de Parkia biglobosa (du placeau) mesurés à hauteur d'homme (1,30 m du sol). Elle se détermine par la formule :

$\mathrm{G}=\sum_{i=1}^{n} c_{i}^{2} / 4 \pi \quad$ avec $\mathrm{G}$, la surface terrière des arbres du placeau et $c_{i}$, la circonférence à hauteur d'homme de l'arbre i du placeau considéré. 
La densité des arbres de Parkia biglobosa est le nombre de pieds de Parkia biglobosa recensés à l'hectare.

Etude de la structure en diamètre des populations de Parkia biglobosa de la zone d'étude

Les pieds de Parkia biglobosa échantillonnés dans chaque commune d'étude ont été répartis par classes de diamètre d'amplitude $10 \mathrm{~cm}$. Des histogrammes traduisant la répartition par classe de diamètre des populations de Parkia biglobosa de chaque commune d'étude ont été tracés à l'aide du tableur Excel.

Etude de la répartition spatiale des populations de Parkia biglobosa

La structure spatiale ou structure au sol des pieds de néré de chaque phytocoenose a été déterminée. La répartition spatiale des populations de Parkia biglobosa a été appréciée au moyen de l'indice de Green $(I G)$ qui est une version améliorée de l'indice de Blackman. Il se formule de la manière suivante (Green, 1966) :

$$
\begin{aligned}
& \mathrm{IG}=\frac{\left(\frac{\delta^{2}}{\mu}\right)-1}{n-1}=\frac{I B-1}{n-1}(3) \\
& I B=\frac{\delta^{2}}{\mu} \text { est l'indice de dispersion }
\end{aligned}
$$

de Blackman (Blackman, 1942) tandis que $\delta$ et $\mu$ sont respectivement l'écart type et la moyenne de la densité de Parkia biglobosa dans la phytocoenose.

Green (1966) a signalé que les valeurs d'IG varient de -1 à +1 , ce qui indique que le mode de répartition spatiale varie d'extrêmement aléatoire à extrêmement agrégé.

L'avantage de l'indice de Green est qu'il peut être utilisé pour comparer des échantillons de populations dont le nombre total d'individus, la moyenne et le nombre d'unités d'échantillonnage varient.

\section{Traitement et analyse des données phytosociologiques}

Après l'encodage des données à partir du tableur Excel, l'individualisation des objets phytosociologiques a été réalisée par une Analyse Factorielle des Correspondances (AFC) et une Classification Hiérarchique Ascendante (CHA) à l'aide du logiciel STATISTICA.

La diversité floristique des milieux étudiés a été appréciée au moyen de la richesse spécifique et des indices de diversité : indice de Shannon et coefficient d'équitabilité de Pielou. La richesse spécifique est le nombre d'espèces présentes dans chaque synusie. L'indice de Shannon-Wiener (H') est calculé sur la base des recouvrements moyens des espèces au sein des relevés. Il est donné par la formule suivante : $H^{\prime}=-\Sigma P_{i} \log _{2} P_{i}(4)$ avec $P_{i}=r_{i} / r(5)$ où $r_{i}$ est le recouvrement de l'espèce i dans le relevé considéré et $r$ désigne la somme totale des recouvrements des espèces du relevé.

Le coefficient d'équitabilité de Pielou (E) est donné par la relation suivante : $\mathrm{E}=$ $\mathrm{H}^{\prime} / \mathrm{H}^{\prime}{ }_{\text {max }}$ (6) avec $\mathrm{H}_{\text {max }}^{\prime}=\log _{2} \mathrm{~S}$ (7) où $\mathrm{S}$ désigne le nombre total d'espèces. $E$ traduit le degré de diversité atteint par rapport au maximum possible. Il est compris entre 0 et 1 . E tend vers 0 lorsqu'il n'y a qu'une seule espèce par relevé. Il prend la valeur 1 lorsque toutes les espèces ont le même recouvrement. 


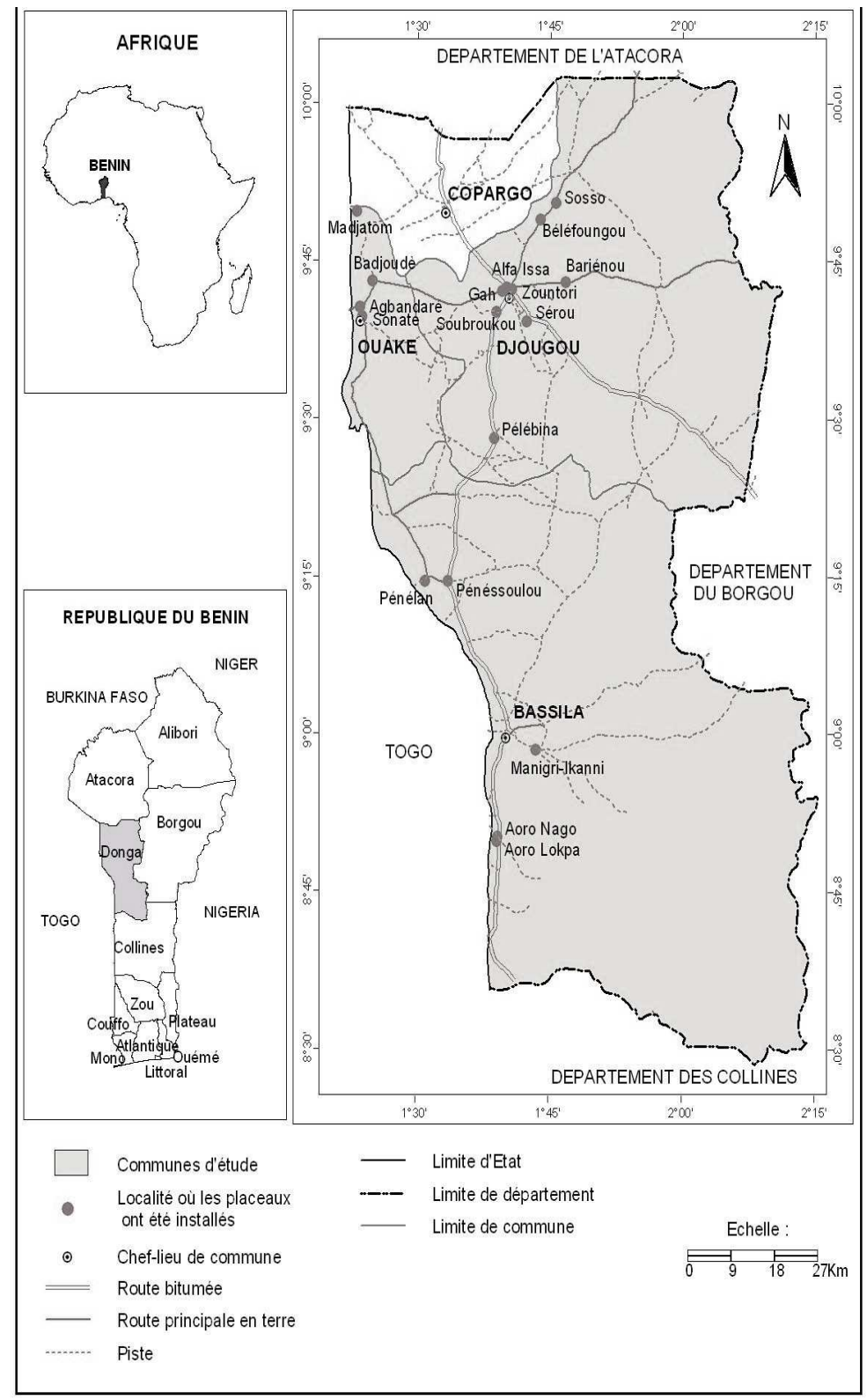

Figure 1: Carte du département de la Donga situant les localités où les placeaux ont été installés.

RESULTATS

Caractéristiques floristiques des différentes synusies identifiées dans les parcs à néré du département de la Donga

L'étude phytosociologique de la végétation spontanée des parcs à néré du département de la Donga a conduit à l'identification de quinze (15) synusies végétales dont les caractéristiques floristiques sont résumées au Tableau 1. Le nombre total d'espèces des différentes synusies obtenues varie de 2 à 105 . Le nombre moyen d'espèces par relevé varie de 1 à 41 . Pour ce qui concerne la diversité spécifique des milieux étudiés, l'indice de Shannon (H') est compris entre 0,02 à 5,08 . Le coefficient d'équitabilité de Pielou (E) varie quant à lui de 0,02 à 0,76 . 
Caractéristiques dendrométriques des phytocoenoses identifiées dans les parcs à néré du département de la Donga

Sur la base de leur interdépendance, les synusies identifiées ont été intégrées en cinq phytocoenoses : la phytocoenose à Pennisetum pedicellatum Trin. ; la phytocoenose à Imperata cylindrica (L.) P. Beauv. et Andropogon gayanus Kunth var.; la phytocoenose à Daniellia oliveri (Rolfe) Hutch. et Dalz.; la phytocoenose à Daniellia oliveri et Pteleopsis suberosa Engl. et Diels; la phytocoenose à Hymenocardia acida Tul. et Terminalia avicennioides Guill. et Perr.. Le Tableau 2 présente les caractéristiques dendrométriques de ces différentes phytocoenoses. Leur densité moyenne varie de 39 pieds/ha à 70 pieds/ha. La surface terrière moyenne quant à elle varie de 4,93 $\mathrm{m}^{2} /$ ha à $12,38 \mathrm{~m}^{2} /$ ha. Le diamètre $\left(D_{\mathrm{g}}\right) \mathrm{de}$ l'arbre de surface terrière moyenne varie de $33,46 \mathrm{~cm}$ à $58,88 \mathrm{~cm}$. Le Tableau 2 rend également compte des résultats de l'analyse de variance simple effectuée pour comparer les différentes phytocoenoses décrites. Cette analyse de variance a été faite en appliquant le test de Newman-Keuls sur les valeurs moyennes des paramètres dendrométriques calculés pour chaque phytocoenose. Elle a permis de faire ressortir les ressemblances et dissemblances qui existent entre ces différentes associations. Toutes les phytocoenoses sont analogues du point de vue de leur densité et de leur surface terrière $(\mathrm{p}$ respectivement égal à 0,688 et 0,127 donc supérieur à 0,05 ). Nous concluons que ces paramètres ne sont donc pas des facteurs discriminatoires pour ces phytocoenoses. En ce qui concerne le diamètre de l'arbre de surface terrière moyenne $\left(\mathrm{D}_{\mathrm{g}}\right)$, la phytocoenose à Imperata cylindrica et Andropogon gayanus est significativement différente des autres au seuil de 5\% ( $p=0,006$ donc $\mathrm{p}<0,05)$. Il est donc certain que le diamètre $D_{g}$ varie en fonction des phytocoenoses et que l'association à Imperata cylindrica et Andropogon gayanus serait favorable au développement du diamètre des pieds de Parkia biglobosa.

Les valeurs d'indice de Green calculées pour caractériser la structure spatiale des pieds de Parkia biglobosa des cinq (5) phytocoenoses sont de 0,023 pour la phytocoenose à Pennisetum pedicellatum; 0,069 pour la phytocoenose à Imperata cylindrica et Andropogon gayanus ; 0,073 pour la phytocoenose à Daniellia oliveri ; 0,007 pour la phytocoenose à Daniellia oliveri et Pteleopsis suberosa; 0,021 pour la phytocoenose à Hymenocardia acida et Terminalia avicennioides. Ces valeurs indiquent que Parkia biglobosa a généralement une distribution de type agrégatif mais parfois il peut présenter une distribution aléatoire.

\section{Structure en diamètre des populations de Parkia biglobosa des communes d'étude}

Les structures en diamètre des populations de Parkia biglobosa dans les communes étudiées (Figures $2 ; 3$ et 4 ) montrent une faible densité des pieds de néré dans les classes inférieures de diamètre surtout dans la classe de diamètre compris entre 10 et $20 \mathrm{~cm}$. A Djougou et à Ouaké, la densité des arbres appartenant à cette classe de diamètre est d'environ 2 pieds par hectare tandis qu'à Bassila elle est d'environ 7 pieds par hectare. Dans les classes de diamètre compris entre 20 et $30 \mathrm{~cm}, 30$ et $40 \mathrm{~cm}, 40$ et $50 \mathrm{~cm}$, la densité en pieds de néré est plus élevée que dans les autres classes de diamètre. Dans ces classes de diamètre, la densité varie entre 12 et 15 pieds de néré par hectare à Bassila, 15 et 19 pieds de néré par hectare à Ouaké, 4 et 6 pieds de néré par hectare à Djougou. Dans la commune de Djougou particulièrement, la structure en diamètre des populations de Parkia biglobosa montre que la densité maximale en pieds de néré ne dépasse pas 6 pieds par hectare. 
Tableau 1: Caractéristiques floristiques des différentes synusies identifiées dans les parcs à néré du département de la Donga.

\begin{tabular}{|c|c|c|c|c|}
\hline Synusies & $\begin{array}{l}\text { Nombre } \\
\text { d'espèces }\end{array}$ & $\begin{array}{c}\text { Nombre moyen } \\
\text { d'espèces par relevé }\end{array}$ & $\begin{array}{l}\text { Indice de diversité } \\
\text { de Shannon (bits) }\end{array}$ & $\begin{array}{c}\text { Indice d'équitabilité } \\
\text { de Pielou }\end{array}$ \\
\hline Synusie annuelle à Pennisetum pedicellatum & 36 & $13(3)$ & $2,47(0,10)$ & $0,48(0,02)$ \\
\hline Synusie annuelle à Pennisetum pedicellatum et Hyparrhenia involucrata & 22 & $4(2)$ & $2,75(0,16)$ & $0,62(0,04)$ \\
\hline Synusie annuelle à Pennisetum pedicellatum et Mitracarpus villosus & 58 & $21(4)$ & $3,78(0,11)$ & $0,65(0,02)$ \\
\hline Synusie vivace basse à Daniellia oliveri & 54 & $10(5)$ & $3,11(0,09)$ & $0,54(0,02)$ \\
\hline Synusie vivace basse à Daniellia oliveri et Ficus sur & 69 & $24(7)$ & $4,38(0,09)$ & $0,72(0,01)$ \\
\hline Synusie vivace basse à Daniellia oliveri et Vitellaria paradoxa & 76 & $24(6)$ & $3,37(0,09)$ & $0,54(0,01)$ \\
\hline Synusie herbacée vivace basse à Stachytarpheta indica & 22 & $2(1)$ & $2,57(0,12)$ & $0,58(0,02)$ \\
\hline Synusie herbacée vivace basse à Stachytarpheta indica et Echinops longifolius & 15 & $3(2)$ & $2,39(0,13)$ & $0,6(0,03)$ \\
\hline Synusie herbacée vivace haute à Andropogon tectorum et Siphonochilus aethiopicus & 13 & $4(2)$ & $1,49(0,13)$ & $0,40(0,03)$ \\
\hline Synusie herbacée vivace haute à Imperata cylindrica et Andropogon gayanus & 14 & $2(1)$ & $0,94(0,15)$ & $0,25(0,06)$ \\
\hline Synusie arbustive à Daniellia oliveri & 30 & $7(4)$ & $2,69(0,1)$ & $0,54(0,02)$ \\
\hline Synusie arbustive à Daniellia oliveri et Pteleopsis suberosa & 86 & $25(8)$ & $4,03(0,08)$ & $0,63(0,01)$ \\
\hline Synusie arbustive à Hymenocardia acida et Terminalia avicennoides & 105 & $41(5)$ & $5,08(0,07)$ & $0,76(0,01)$ \\
\hline Synusie arborescente à Parkia biglobosa & 2 & $1(1)$ & $0,02(0,01)$ & $0,02(0,01)$ \\
\hline Synusie arborescente à Parkia biglobosa et Vitellaria paradoxa & 18 & $3(2)$ & $1,7(0,16)$ & $0,41(0,04)$ \\
\hline
\end{tabular}

N.B. : Les chiffres entre parenthèses représentent les écart-types calculés. 
Tableau 2 : Caractéristiques dendrométriques des phytocoenoses identifiées dans les parcs à néré du département de la Donga.

\begin{tabular}{|c|c|c|c|}
\hline Phytocoenoses & $\begin{array}{l}\text { Densité moyenne } \\
\text { (pieds/ha) }\end{array}$ & $\begin{array}{l}\text { Surface terrière moyenne } \\
\qquad\left(\mathbf{m}^{2} / \mathbf{h a}\right)\end{array}$ & $\begin{array}{l}\text { Diamètre moyen Dg } \\
(\mathbf{c m})\end{array}$ \\
\hline Phytocoenose à Pennisetum pedicellatum & $70 \mathrm{~A}(23)$ & $10,89 \mathrm{~A}(4,41)$ & $44,34 \mathrm{~A}(5,61)$ \\
\hline Phytocoenose à Imperata cylindrica et Andropogon gayanus & $51 \mathrm{~A}(28)$ & $12,38 \mathrm{~A}(6,50)$ & $58,88 \mathrm{~B}(13,33)$ \\
\hline Phytocoenose à Daniellia oliveri & $53 \mathrm{~A}(39)$ & $6,74 \mathrm{~A}(4,93)$ & $40,50 \mathrm{~A}(5,59)$ \\
\hline Phytocoenose à Daniellia oliveri et Pteleopsis suberosa & 39A (3) & $4,93 \mathrm{~A}(1,63)$ & $39,78 \mathrm{~A}(5,90)$ \\
\hline $\begin{array}{l}\text { Phytocoenose à Hymenocardia acida et Terminalia } \\
\text { avicennioides }\end{array}$ & $50 \mathrm{~A}(14)$ & $4,96 \mathrm{~A}(3,77)$ & $33,46 \mathrm{~A}(9,51)$ \\
\hline
\end{tabular}

Les chiffres entre parenthèses sont les écart-types. 


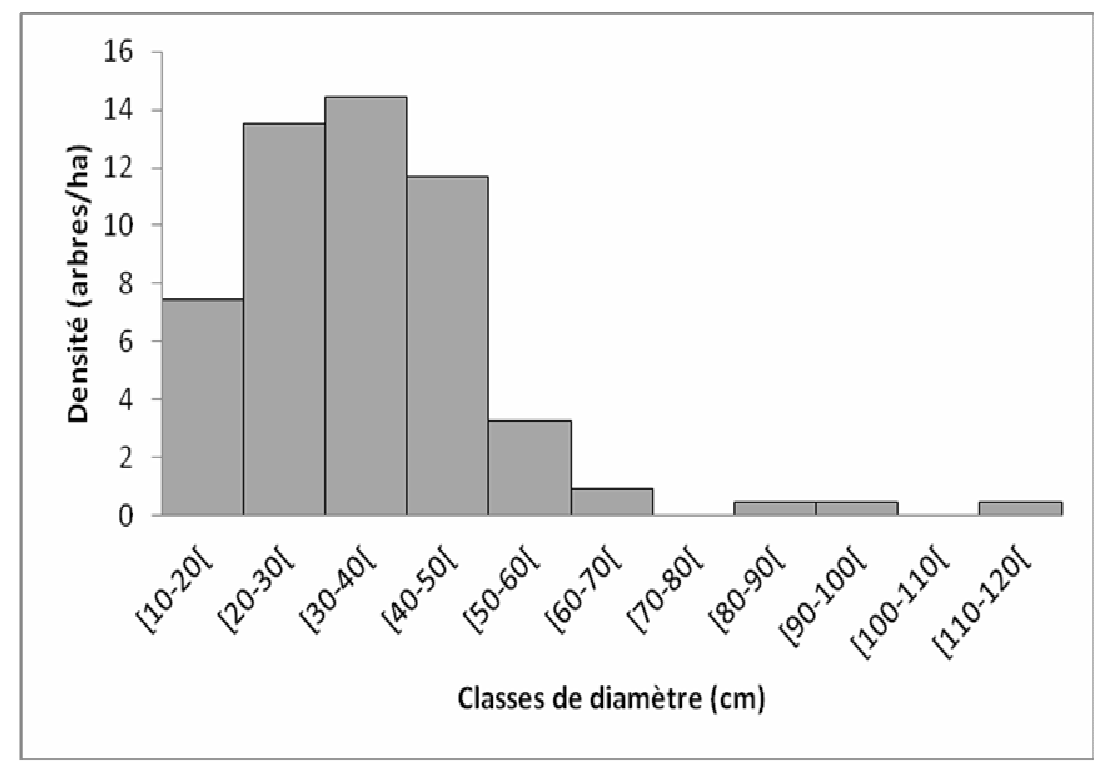

Figure 2: Structure en diamètre des populations de Parkia biglobosa de la commune de Bassila.

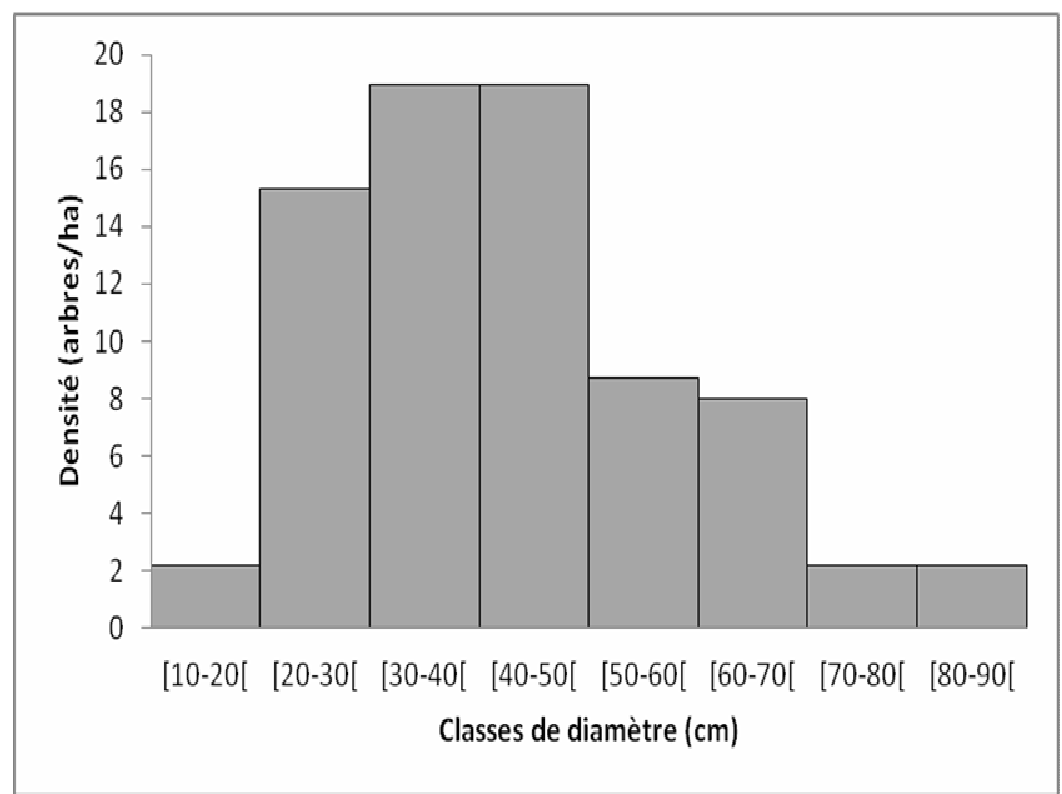

Figure 3 : Structure en diamètre des populations de Parkia biglobosa de la commune de Ouaké. 


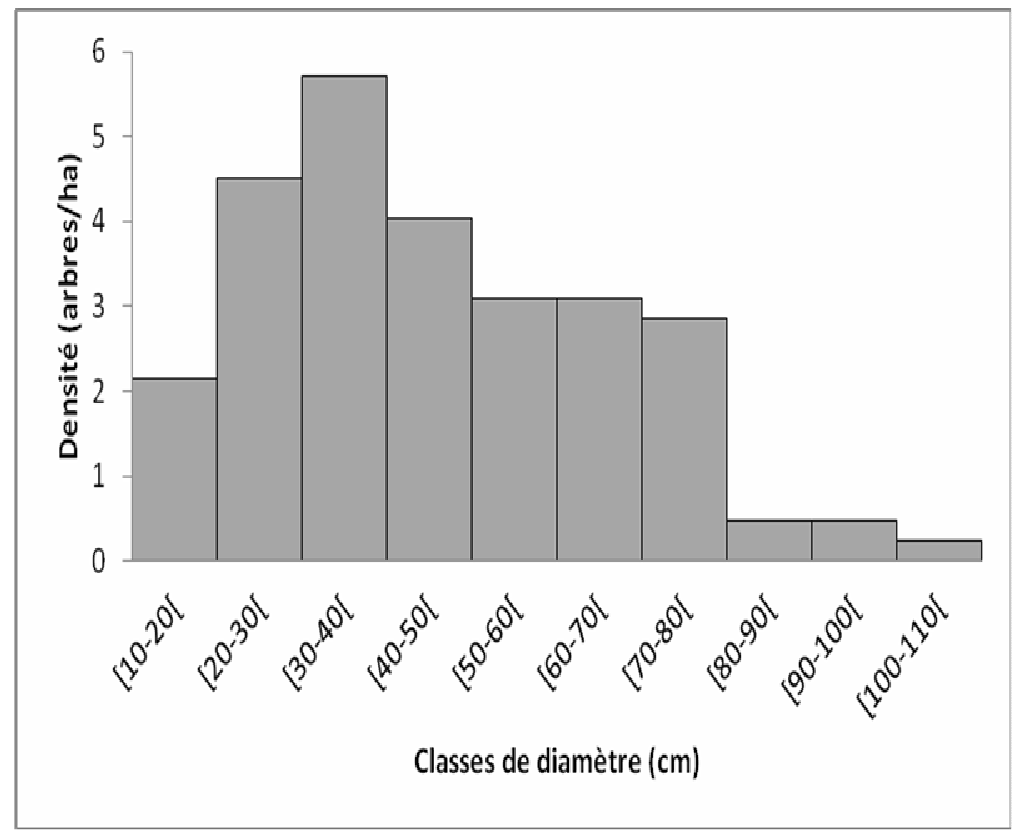

Figure 4 : Structure en diamètre des populations de Parkia biglobosa de la commune de Djougou.

\section{DISCUSSION}

Paramètres phytosociologiques et
dendrométriques des peuplements
Parkia biglobosa
Pour mieux appréhender la dégradation des écosystèmes qui est d'ordre naturel et anthropique, l'étude de la végétation paraît très indiquée. En effet, la végétation d’une région est le résultat d'un long processus de sélection naturelle sous l'action du climat, des conditions édaphiques et topographiques, sans oublier les activités anthropiques (Adedire, 2002 ; Orthmann, 2005 ; Parmentier et al., 2005). Les résultats de la présente étude ont une fois encore souligné l'utilité de la phytosociologie comme outil efficace d'investigation de la végétation.

Les valeurs de l'indice de Shannon et du coefficient d'équitabilité de Pielou obtenues pour les différentes synusies de la présente étude montrent une structure spécifique assez diversifiée.
Les différentes espèces de chaque synusie sont inégalement représentées en termes d'individus et de recouvrement. Le même constat a été fait par Ganglo (2004) dans le périmètre forestier de Toffo.

Une faible richesse spécifique est notée au niveau de la synusie arborescente à Parkia biglobosa. Cette faible richesse spécifique constatée au niveau de cette synusie arborescente s'explique par le fait que Parkia biglobosa est généralement seul ou accompagné de Vitellaria paradoxa et ils constituent tous deux les principales composantes de la strate arborée. Ceci est la conséquence de la gestion sélective de la végétation par les paysans et qui consiste à préserver seulement les arbres « utiles » aux hommes. Les autres synusies sont par contre accompagnées d'une variété d'espèces. Cela est plus remarquable lorsqu'on considère la synusie arbustive à Hymenocardia acida et Terminalia avicennioides qui affiche la plus grande valeur de l'indice de Shannon (5,08 bits) et du coefficient d'équitabilité de Pielou $(0,76)$. 
Une grande richesse spécifique est notée au niveau de cette synusie (105 espèces). Par opposition aux milieux colonisés par les espèces des synusies herbacées et des synusies arborescentes, la synusie arbustive à Hymenocardia acida et Terminalia avicennioides abritent des peuplements plus ou moins équilibrés où les conditions sont favorables à l'installation plus ou moins équitable de nombreuses espèces.

Les valeurs de densité moyenne obtenues pour les phytocoenoses identifiées dans la présente étude (39 à 70 pieds/ha) sont supérieures à celles obtenues par Gbédji (2003) au niveau des parcs à Parkia biglobosa des régions de Bohicon, Savè, Parakou, Bembérèkè et Kandi (1 à 5 pieds/ha). Ces faibles valeurs obtenues par Gbédji (2003) peuvent être dues au fait que les pieds de Parkia biglobosa de la plupart de ces parcs soient soumis à une forte pression anthropique. Selon cet auteur, au niveau des parcs à Parkia biglobosa de la région de Bohicon, les pieds de Parkia biglobosa sont abattus et utilisés dans l'artisanat pour fabriquer des mortiers et pilons. En ce qui concerne la région de Savè, la population s'est spécialisée dans la vente du charbon de bois et de bois de feu et les pieds de Parkia biglobosa sont parmi les espèces victimes de cette carbonisation.

Les valeurs de densité moyenne obtenues pour les phytocoenoses identifiées dans la présente étude (39 à 70 pieds/ha) sont également supérieures à celles obtenues par Ouédraogo (1995) dans différentes zones du Burkina Faso (5 à 25 pieds/ha). Selon cet auteur, les zones où la densité de Parkia biglobosa est élevée sont des zones où l'espèce a une grande importance socioéconomique et culturelle dans la vie des populations. Ceci est le cas dans notre zone d'étude (le département de la Donga) où les connaissances locales sur Parkia biglobosa sont diversifiées et varient en fonction de l'ethnie, de l'âge et du sexe (Koura et al., 2011).

La connaissance de la surface terrière du peuplement est d'un intérêt très pratique et est souvent recommandée en sylviculture. Souvent, influencée par les traitements sylvicoles pratiqués, la fixation de sa valeur détermine l'intensité des interventions lors des aménagements forestiers (Sokpon, 1995). La comparaison de la surface terrière moyenne des pieds de Parkia biglobosa se trouvant dans chacune des phytocoenoses étudiées montre des valeurs plus ou moins faibles de surface terrière moyenne (4,93 à 12,38 $\left.\mathrm{m}^{2} / \mathrm{ha}\right)$. Les pieds de néré de la phytocoenose à Imperata cylindrica et Andropogon gayanus ont la surface terrière moyenne la plus élevée $\left(12,38 \mathrm{~m}^{2} / \mathrm{ha}\right)$ et leur diamètre moyen $\mathrm{D}_{\mathrm{g}}$ est le plus élevé $(58,88 \mathrm{~cm})$. L'association à Imperata cylindrica et Andropogon gayanus serait alors favorable au développement $\mathrm{du}$ diamètre et de la surface terrière des pieds de Parkia biglobosa.
Structure en diamètre et répartition spatiale des peuplements de Parkia biglobosa de la zone d'étude

L'étude du regroupement des tiges par catégories de grosseur présente un grand intérêt en matière de gestion forestière car elle est une expression de la «structure» du peuplement (Rondeux, 1999). Elle traduit aussi la réaction de celui-ci aux conditions de croissance et aux opérations sylvicoles pratiquées. Dans la zone d'étude, les pieds de Parkia biglobosa font généralement partie du paysage agricole. Ainsi, les structures en diamètre des populations de Parkia biglobosa traduisent l'impact de la gestion des parcs à néré par les paysans. D'après nos résultats, cette gestion paysanne ne se soucie pas de la régénération de l'espèce et celle-ci présente un net déficit dans les classes inférieures de diamètre surtout dans la classe de diamètre compris entre 10 et $20 \mathrm{~cm}$. Ainsi, en matière de gestion des populations de néré dans le département de la Donga ces structures en diamètre montrent une insuffisance dans les mesures de régénération du néré. Si des dispositions ne sont pas prises, à terme l'utilisation durable de l'espèce sera remise en cause. Il est donc indispensable pour l'utilisation durable des ressources en néré du département, de penser à l'enrichissement des parcs en néré surtout les parcs de la commune de Djougou où la structure en diamètre des populations de Parkia biglobosa montre que la densité maximale en pieds de néré ne dépasse pas 6 pieds par hectare.

Les valeurs de l'indice de Green montrent une distribution de type agrégatif du néré. Ces valeurs $(0,021 ; 0,023 ; 0,069 ; 0,073)$ traduisent un regroupement relativement faible de ces pieds, ce qui concorde bien avec les observations faites sur le terrain. En ce qui concerne la phytocoenose à Daniellia oliveri et Pteleopsis suberosa, la valeur de l'indice de Green ($0,007)$ montre que la dispersion des pieds de Parkia biglobosa de cette phytocoenose est très aléatoire. Selon les observations du terrain, les pieds de Parkia biglobosa de cette phytocoenose forment une synusie arborescente éclatée en fragments réduits à quelques individus. Manish et Sundaramoorthy (2008), étudiant la distribution spatiale de l'espèce Corchorus depressus dans le désert semi-aride indien, ont trouvé des valeurs d'indice de Green variant de $-0,2$ à 0 . Ils ont conclu que ces valeurs indiquent une distribution aléatoire de l'espèce Corchorus depressus.

\section{Mesures préconisées pour l'aménagement et la gestion durable des parcs à néré du département de la Donga}

Les parcs à néré du départerment de la Donga sont gérés par les propriétaires de ces pieds de néré. Aucun plan d'aménagement préalablement établi n'existe. Dans la zone d'étude, les pieds de Parkia biglobosa font généralement partie du paysage agricole. Malgré la grande importance socio-économique et culturelle de Parkia biglobosa dans la vie des populations de ce 
département (Koura, 2003), Parkia biglobosa est soumis à des actions anthropiques telles que les feux de brousse, les prélèvements d'organes à des fins médicinales, la fabrication de charbon, le pâturage, etc. Sadji (2000) avait déjà noté dans certains terroirs villageois de la commune de Djougou (Nalohou, Sassero, Soubroukou, Wargou) que les feux qui passent chaque année ralentissent et entravent le développement des essences floristiques parmi lesquelles Parkia biglobosa figure.

$\mathrm{Au}$ vu de tout ce qui précède, des mesures doivent donc être prises pour l'aménagement et la gestion durable des parcs à néré de ce département. L'administration forestière devrait plus s'intéresser à la gestion de ces parcs. A moyen et à long terme, on devrait penser à l'encadrement des populations pour la mise en place de vastes plantations de Parkia biglobosa et l'enrichissement des parcs par introduction des individus produits en pépinière. Ceci est important car tous les organes de Parkia biglobosa sont largement valorisés pour la satisfaction des besoins de l'homme (Ouédraogo, 1995). De plus, Parkia biglobosa fait partie de la famille des Fabacées, famille de plantes reconnues pour leur grand rôle dans l'amélioration de la fertilité des sols. Les études sur la sylviculture de l'espèce devraient donc être approfondies.

\section{Conclusion}

L'application de la phytosociologie synusiale à l'étude de la végétation spontanée autour des pieds de néré du département de la Donga a révélé les différents niveaux d'organisation de cette végétation. Celle-ci se compose de quinze (15) synusies végétales réparties en cinq (5) phytocoenoses sur la base de leurs relations spatio-temporelles : la phytocoenose à Pennisetum pedicellatum ; la phytocoenose à Imperata cylindrica et Andropogon gayanus; la phytocoenose à Daniellia oliveri; la phytocoenose à Daniellia oliveri et Pteleopsis suberosa et la phytocoenose à Hymenocardia acida et Terminalia avicennioides.

Les différents paramètres écologiques et dendrométriques déterminés dans la présente étude ont permis d'avoir des informations précieuses sur les pieds de Parkia biglobosa retrouvés dans ces phytocoenoses. Ces informations doivent être complétées afin de pouvoir prendre de bonnes décisions d'aménagement et de gestion durables des parcs à néré de la zone d'étude. Aussi, nous suggérons: (1) la mise en place de placeaux permanents pour un suivi de la dynamique de cette espèce ; (2) la collecte périodique de données sur ces placeaux pour élaborer à terme, les normes de sylviculture de cette espèce.

\section{REMERCIEMENTS}

Nous remercions l'Université d'Abomey-Calavi à travers le projet de fonds compétitifs «Néré 2ADE » pour le financement de ce travail.

\section{REFERENCES}

Adedire MO. 2002. Environmental implications of tropical deforestation. J. Sustain. Dev. World Ecol., 9: $33-40$.

Azokpota P, Hounhouigan DJ, Nago MC. 2006. Microbiological and chemical changes during the fermentation of African locust bean (Parkia biglobosa) to produce afitin, iru and sonru, three traditional condiments produced in Benin. International Journal of Food Microbiology, 107: 304-309.

Baco MN. 2007. Gestion locale de la diversité cultivée au Nord Bénin : éléments pour une politique publique de conservation de l'agrobiodiversité de l'igname (Dioscorea spp.). Thèse de doctorat en Socio-anthropologie, Université d'Orléans, p. 406.

Bikoué MAC, Essomba H. 2007. Gestion des ressources naturelles fournissant les Produits Forestiers Non Ligneux alimentaires en Afrique Centrale. Produit Forestier Non ligneux. Document de Travail N ${ }^{\circ} 5$. Organisation des Nations Unies pour l'Alimentation et l'Agriculture, p. 103.

Blackman GE. 1942. Statistical and Ecological Studies in the Distribution of Species in Plant Communities: I. Dispersion as a Factor in the Study of Changes in Plant Populations. Ann. Bot., 6(2): 351-370.

Bonkoungou EG. 1987. Monographie du néré, Parkia biglobosa (jacq.) Benth., espèce agroforestière à usages multiples. Institut de recherche en biologie et écologie tropicale. IRBET / CNRST, Ouagadougou, Burkina Faso, p. 42.

Codjia JTC, Assogbadjo AE, Ekué MRM. 2003. Diversité et Valorisation au Niveau Local des Ressources Végétales Forestières Alimentaires du Bénin. Cahiers d'Etudes et de Recherches Francophones / Agricultures, 12(5): 321-331.

Djakpo O. 2005. Fermentation contrôlée des graines de néré (Parkia biglobosa) pour la production d'un condiment béninois de type afitin : effets de l'utilisation des souches sélectionnées de Bacillus subtilis sur la qualité du produit. Thèse d'ingénieur Agronome, Université d'Abomey-Calavi, p. 72.

Duplat P, Perrote G. 1981. Inventaire et estimation de l'accroissement des peuplements forestiers. Office National des Forêts. Section technique, p. 432.

Eyog-Matig O, Adjanohoun E, de Souza S, Sinsin B. 1999. Réseau «Espèces Ligneuses Médicinales». Compte rendu de la première réunion du Réseau, tenue les 15-17 décembre 1999 à la Station IITA Cotonou, Bénin. Institut International des Ressources Phytogénétiques, p. 141. 
Eyog-Matig O, Gaoué OG, Dossou B. 2002. Réseau « Espèces Ligneuses Alimentaires ». Compte rendu de la première réunion du Réseau tenue 11-13 décembre 2000 au CNSF Ouagadougou, Burkina Faso. Institut International des Ressources Phytogénétiques, P. 235 + annexe.

FAO 1999. State of the world's forests. Doc. FAO, p. 154.

Ganglo CJ. 2004. Phytosociologie appliquée à l'aménagement des forêts: cas du périmètre forestier de Toffo (Sud Bénin, Département de l'Atlantique). Rapport de travail. Faculté des Sciences Agronomiques de l'Université d'Abomey-Calavi. Département d'Aménagement et Gestion de l'Environnement, p. 241.

Ganglo CJ, de Foucault B. 2006. Plant communities, forest site identification and classification in Toffo reserve, South-Benin. Bois et Forêts des Tropiques. 288(2): 25-38.

Gbédji EK. 2003. Caractérisation morphologique et structurale des parcs à néré (Parkia biglobosa (Jack.) R. Br. Ex. G. Don.) au Bénin. Thèse d'Ingénieur Agronome, FSA /UNB, p. $109+$ annexes.

Gillet F, Foucault (de) B, Julve P. 1991. Phytosociologie synusiale intégrée : objets et concepts. Candollea, 46(2): 315-340.

Gillet F. 2000. Phytosociologie Synusiale Intégrée. Guide Méthodologique (4 ${ }^{\mathrm{ème}}$ édn revue et corrigée). Université de Neuchâtel - Institut de Botanique; p. 68.

Green RH. 1966. Measurement of non-randomness in spatial distributions. Res. Pop. Ecol., 8: 1-7.

Gutierrez ML. 2000. Production et Commercialisation de l'Afitin Fon dans la Région d'Abomey-Bohicon au Bénin. CIRAD, p. 124.

INSAE. 2004. Cahier des Villages et Quartiers de ville. Département de la Donga. Direction des Etudes Démographiques : Cotonou, Bénin ; 15.

Koura K. 2003. Contribution à l'étude ethnobotanique du néré [Parkia biglobosa (Jacq.) R. Br. ex G. Don] dans les départements de l'Atacora et de la Donga : Aspects socioculturels. Mémoire de DESS en Aménagement et Gestion des Ressources Naturelles option Sciences et Techniques Forestières. FSA / UAC, p. 94 + annexes.

Koura K, Ganglo CJ, Assogbadjo AE, Agbangla C. 2011. Ethnic differences in use values and use patterns of Parkia biglobosa in Northern Benin. Journal of Ethnobiology and Ethnomedicine, 7: 42.

Lafleur M. 2008. Recherches et Documentation des Meilleures Pratiques pour la Gestion Durable des Parcs en Karité en Afrique de l'Ouest. CECI: Montréal; 110.
Manish M, Sundaramoorthy S. 2008. Distribution pattern and growth assessment of Corchorus depressus in semi arid Indian desert. Tropical Ecology, 49(1): 6971.

Mukerji AK. 1995. Mémoire spécial sur l'importance des produits forestiers non ligneux et des stratégies de développement durable. Produits Forestiers non Ligneux, 15: 225 - 237.

Orthmann B. 2005. Vegetation ecology of a woodlandsavanna mosaic in central Benin (West Africa): Ecosystem analysis with a focus on the impact of selective logging Dissertation. University of Rostock, p. 148.

Ouédraogo A-S. 1995. Parkia biglobosa (Leguminosae) en Afrique de l'Ouest : biosystématique et amélioration. Thèse de Doctorat, Université Agronomique de Wageningen, p. 205.

Parmentier I, Stevart T, Hardy OJ. 2005. The inselberg flora of Atlantic Central Africa. I. Determinants of species assemblages. Journal of Biogeography, 32: $685-696$.

Rondeux J. 1999. La Mesure des Peuplements Forestiers. Presses agronomiques de Gembloux: Gembloux; 522.

Sadji B. 2000. Contribution à la promotion du néré (Parkia biglobosa) dans les terroirs villageois de la Circonscription Urbaine de Djougou: Cas des villages de Nalohou, Sassero, Soubroukou, Wargou. Mémoire de fin de cycle pour l'obtention du Brevet de Technicien Supérieur, Ecole de Spécialisation en Foresterie du Banco, République de Côte d'Ivoire, p. 54.

Sina S. 2006. Reproduction et diversité génétique chez Parkia biglobosa (Jacq.) G. Don. PhD thesis, Wageningen University, p. 102.

Sokpon N. 1995. Recherche écologique sur la forêt dense semi-décidue de Pobè au sud-Bénin : groupements végétaux, structure, régénération naturelle et chute de litière. Thèse de Doctorat, Université Libre de Bruxelles, p. 350.

Walla K. 2001. Typologie, structure et fonctionnement des agrosystèmes traditionnels dans la Préfecture de Doufélgou (Nord-Togo). Mémoire de DESS en Aménagement et Gestion des Ressources Naturelles, FSA / UAC, p. $72+$ annexes.

Wong JLG, Thornber K, Baker N. 2001. Évaluation des ressources en produits forestiers non ligneux : expériences et principes de biométrie. Rome, Italie. Produits Forestiers non Ligneux, 13: 108. 